\title{
Limitations of ventricular pacing maneuvers to differentiate orthodromic reciprocating tachycardia from atrioventricular nodal reentry tachycardia
}

\author{
Péter Kupó $^{1}\left[\right.$ C Cristina loana Tutuianu $^{1} \cdot$ Genadi Kaninski $^{1} \cdot$ Zoltán Gingl $^{1} \cdot$ László Sághy $^{1} \cdot$ Róbert Pap $^{1}$
}

Received: 5 November 2020 / Accepted: 7 April 2021

(C) Springer Science+Business Media, LLC, part of Springer Nature 2021

\begin{abstract}
Purpose Various ventricular pacing maneuvers have been developed to differentiate orthodromic reciprocating tachycardia (ORT) from atrioventricular nodal reentry tachycardia (AVNRT). We aimed to evaluate the diagnostic value of ventricular pacing maneuvers in patients undergoing catheter ablation for AVNRT/ORT.

Methods Sixty patients with supraventricular tachycardia (SVT) undergoing invasive EP study were included (ORT: 31, typical AVNRT: 18, atypical AVNRT: 11). Ventricular overdrive pacing (VOP) and resetting by premature ventricular stimulation (PVS) during SVT were analyzed by 3 independent observers blinded to the ultimate diagnosis. We determined intraclass correlation coefficient (ICC) for interobserver agreement and the diagnostic accuracy of consensual results.

Results Although specificity of all parameters was high (96-100\%) for ORT, semi-quantitative parameters of VOP (requiring the recognition of specific ECG patterns) had lower interobserver reliability (ICC: $0.32-0.66$ ) and sensitivity (16.1-77.4\%). In contrast, most quantitative measurements of VOP and PVS showed good reproducibility (ICC: 0.93-0.95) and sensitivity (74.2-89.3\%), but post-pacing interval after VOP needed correction with AV nodal conduction slowing. False negative results for diagnosing ORT were more common with left free wall vs. right free wall or septal, and slowly vs. fast-conducting septal APs. False positivity was only seen with a bystander, concealed nodo-fascicular/nodo-ventricular (NF/NV) AP in a case of AVNRT. Conclusions No single maneuver is $100 \%$ sensitive for ORT. Semi-quantitative features have limited reproducibility and all parameters can be misleading in the case of rate-dependent delay during VOP/PVS, ORT circuits remote from the pacing site, or a bystander, concealed NF/NV AP.
\end{abstract}

Keywords Paroxysmal supraventricular tachycardia Ventricular pacing maneuvers - Atrioventricular nodal reentrant tachycardia $\cdot$ Atrioventricular reentrant tachycardia $\cdot$ Accessory pathway

\section{Introduction}

Various features and pacing maneuvers are used in the electrophysiology (EP) laboratory to differentiate orthodromic reciprocating tachycardia (ORT) from atrioventricular nodal reentry tachycardia (AVNRT). The atrial activation sequence can be helpful when ORT is mediated by a free wall accessory pathway (AP); however, left and right atrial recordings are not always available. Moreover, concentric coronary sinus (CS)

Péter Kupó

peter.kupo@gmail.com

1 2nd Department of Internal Medicine and Cardiology Centre, Medical School, University of Szeged, Semmelweis u. 8, Szeged H-6725, Hungary activation by a left free wall AP has been reported [1] and an eccentric pattern does not exclude AVNRT.[2] Therefore, further diagnostic maneuvers should be included in the armamentarium to be able to confidently diagnose ORTs involving both septal and free wall APs. These include entrainment and resetting of supraventricular tachycardia (SVT) by ventricular overdrive pacing (VOP) and premature ventricular stimulation (PVS). During these maneuvers, the recognition of surface or intracardiac electrocardiographic patterns (semi-quantitative parameters) or the measurement of certain intervals (quantitative parameters) is used in the differential diagnosis. Most of these maneuvers, however, have only been tested by the group who originally described them and their limitations were highlighted only later by case reports describing their pitfalls.[1, 3-10] While some suggested that a single pacing maneuver could be able to distinguish ORT from AVNRT [11], the optimal parameter in terms of interobserver reliability 
and diagnostic accuracy has not been determined. The aim of this study was to provide an independent evaluation of the diagnostic value and characterize the limitations of ventricular pacing maneuvers in patients undergoing catheter ablation of AVNRT/ORT.

\section{Methods}

We retrospectively studied patients undergoing invasive EP studies for paroxysmal SVT. Patients were included if they had inducible and sustained ORT or AVNRT; the maximum spontaneous oscillation in tachycardia cycle length (TCL) was less than 30 milliseconds $(\mathrm{ms})$ at all times and less than $10 \mathrm{~ms}$ at the time of delivery of the pacing maneuvers, which had to be successfully undertaken. The EP study was performed under conscious sedation using midazolam \pm fentanyl, in a fasting state. All patients gave written informed consent. Antiarrhythmic drugs were discontinued for at least five half-lives prior to the procedure. The protocol of the study is in accordance with the Declaration of Helsinki.

\subsection{EP procedures}

Following femoral venous access, one decapolar steerable catheter (interelectrode spacing 2-5-2 mm, Dynamic Deca, Bard Electrophysiology, Lowell, MA, USA) was positioned in the coronary sinus (CS), one quadripolar electrode catheter in the right ventricular apex (RVA), and one mapping/ablation catheter was positioned in the His bundle region. Twelve lead electrocardiogram (ECG) and intracardiac electrograms were recorded and stored on a digital recording system (CardioLab, GE Healthcare, Chicago, IL, USA), using a band pass filter between 30 and $500 \mathrm{~Hz}$.

\subsection{Pacing maneuvers}

VOP during SVT was performed from the RVA at a cycle length 10-30 ms shorter than the TCL. The onset of pacing was synchronized to the last sensed RVA electrogram. Entrainment was confirmed when the atrial rate accelerated to the pacing cycle length and the tachycardia continued after pacing was stopped.

Resetting of SVT by PVS was attempted by scanning diastole with progressively premature ventricular extrastimuli delivered from the RVA, until resetting/termination of the tachycardia occurred or ventricular refractoriness was reached.

\subsection{Blinded analysis}

Successful attempts of VOP and PVS were analyzed by 3 independent observers blinded to the ultimate diagnosis.
Semi-quantitative parameters analyzed during VOP were the following:

1. Perturbation of atrial rate in the transition zone VOP during SVT produces progressive QRS fusion before the QRS morphology becomes stable. If atrial timing is perturbed or a fixed stimulus-atrial interval is established within this so-called transition zone, ORT is the likely mechanism. The transition zone includes the first entrained QRS with a stable morphology, determined on the 12-lead surface ECG.[6]

2. Ventricular fusion

After the transition zone, during stable entrainment by VOP, the stimulated wavefront collides with the anterograde wavefront of the previous beat. In ORT, this collision can occur in the ventricular myocardium leading to manifest QRS fusion.[7] Stable, entrained, 12-lead QRS morphology was compared to ventricular pacing during sinus rhythm at the same cycle length to determine the presence of fusion during VOP.

3. Atrial reset with or before septal ventricular electrogram (SVE) reset

During VOP, advancement of the atrial electrogram before or immediately with SVE advancement by the paced wave front may predict the diagnosis of the SVT to be ORT.[8]

4. Anterograde His or SVE capture during VOP

To evaluate anterograde His or SVE capture, the first QRS complex after the termination of VOP and its corresponding His bundle electrogram (HBE) were identified. An interval equal to the pacing cycle length was measured back from the HBE. When the preceding, captured HBE occurred at this interval, anterograde activation was confirmed. In the absence of visible, captured HBE, SVE was used as a substitute in the same manner.[5]

Measurements (quantitative parameters) during VOP and PVS with the longest coupling interval that resulted in resetting of SVT included:

1. The difference between stimulus-A and VA (SA-VA) intervals

Stimulus-A interval was measured during VOP from the last pacing stimulus to the earliest atrial activation of the last entrained beat. VA interval was defined as the interval from the onset of the earliest ventricular activation be it on the surface QRS complex or a ventricular electrogram and the earliest atrial electrogram during SVT before VOP. $[9,12]$

2. The post-pacing interval (PPI) minus TCL (PPI-TCL) and corrected PPI-TCL (cPPI-TCL)

PPI was measured from the last pacing stimulus of the VOP/PVS to the earliest ventricular electrogram after the 
VOP/PVS, recorded on the pacing channel.[9] PPI was corrected with the difference between the AH interval following the last entrained beat and the $\mathrm{AH}$ interval of the SVT before VOP/PVS.[10, 12] This parameter was defined as cPPI.

\subsection{His-refractory PVS}

Advancement/delay of the atrial activation with resetting or SVT termination during PVS at His refractoriness was noted. PVS was considered to be His refractory when it occurred no more than $30 \mathrm{~ms}$ earlier than the anticipated timing of the antegrade His electrogram.

\subsection{Diagnosis of SVT}

The diagnosis was established by a combination of standard electrophysiologic criteria, pacing maneuvers, and results of ablation. Diagnostic criteria for AVNRT included an A-(H)-V response after VOP, with an SA-VA interval $>85 \mathrm{~ms}$ and cPPI-TCL $\geq 110 \mathrm{~ms}$, noninducibility of the tachycardia after ablation at the AV nodal slow pathway region resulting in junctional acceleration and elimination or modification of anterograde or retrograde slow pathway conduction. AVNRT was considered to be atypical when the septal VA interval during tachycardia was $>70 \mathrm{~ms}$. Criteria for ORT included an $\mathrm{A}-(\mathrm{H})-\mathrm{V}$ response after VOP, with SA-VA interval < $85 \mathrm{~ms}$ and cPPI-TCL $<110 \mathrm{~ms}$, His-refractory PVS resetting or terminating the tachycardia, an increase in the VA interval during tachycardia by at least $20 \mathrm{~ms}$ with the development of bundle branch block, and ablation at the earliest atrial or ventricular site during retrograde or anterograde AP conduction resulting in elimination of the tachycardia. An AP was defined as slowly conducting if the VA/TCL was $\geq 40 \%$ and, conversely, fast conducting if the VA/TCL was $<40 \%$.

Whenever examining the above criteria gave contradictory results, additional features and maneuvers were studied, including $\mathrm{AH}$ interval during sinus rhythm and tachycardia [13], difference between the HA interval during VOP and SVT $\left(\mathrm{HA}_{\mathrm{VOP}}-\mathrm{HA}_{\mathrm{SVT}}\right)$ [14], pacing closer to the presumed site of the AP (base or outflow tract of the right ventricle, left ventricle) [1], and the para-Hisian pacing maneuver.[15]

\subsection{Statistical analysis}

The results of the three blinded observers were compared by computing intraclass correlation coefficient (ICC) to determine interobserver agreement. In the case of semiquantitative parameters of VOP, any disagreement between observers was resolved by consensus. For the quantitative parameters, the mean of the three values determined by the observers was used for further analysis. Accuracy of each parameter in diagnosing ORT was determined for consensual results. Parametric data are presented as mean and standard deviation. Categorical data are presented as percentage. Data were analyzed according to their normal distribution on the Shapiro goodness-of-fit test. Paired $t$-tests were used for the comparison of normally distributed continuous variables in the same group. Non-normally distributed paired variables were compared using the Wilcoxon matched-pairs test. Comparing independent groups, continuous data were analyzed using one-way ANOVA, followed by post hoc tests and Bonferroni correction. Categorical variables were compared using chi-square test. A $p$ value $<0.05$ was considered statistically significant in all analyses. Statistical analyses were performed using SPSS 24 software (SPSS, Inc., Chicago, IL, USA).

\section{Results}

Sixty patients were included in the study, 31 of them presented with ORT, and 18 with typical and 11 with atypical AVNRT (Table 1). The successful ablation site of the patients with ORT was septal in 12, and on the right in 4 and on the left free wall in 15 cases.

\subsection{Response to VOP}

\subsubsection{Semi-quantitative parameters}

The recognition of the four semi-quantitative features was not consistent between observers, reflected by a low ICC value (Table 2). Even after consensus, they were judged to be missing frequently during VOP of ORT, accounting for their limited sensitivity (Table 2 ).

\subsubsection{Quantitative parameters}

Interobserver agreement was better for quantitative measurements of VOP with a comparably high specificity. Sensitivity was also better compared to the semi-quantitative parameters; however, correcting the PPI for AV nodal delay cannot be neglected (Table 2).

The PPI and cPPI measured after PVS with the longest coupling interval resetting the tachycardia had equally high specificity and better sensitivity compared to their counterpart after VOP (Table 2).

There was no difference in quantitative parameters between SVTs with right free wall and septal APs, but all measurements were significantly longer in the case of left free wall APs (Table 1). 
Table 1 Clinical and tachycardia characteristics of the study population

Typical AVNRT Atypical AVNRT Right free wall ORT Septal ORT Left free wall ORT $p$ value

\begin{tabular}{|c|c|c|c|c|c|c|}
\hline \multicolumn{7}{|l|}{ Demographics } \\
\hline Age, years & $50.6 \pm 20.3$ & $47.3 \pm 19.9$ & $49.8 \pm 23.6$ & $48.9 \pm 17.9$ & $40.5 \pm 18.0$ & 0.71 \\
\hline Male, $\%$ & 44.4 & 27.3 & 50.0 & 77.8 & 60.0 & 0.77 \\
\hline \multicolumn{7}{|l|}{ SVT characteristics } \\
\hline TCL, ms & $359 \pm 61$ & $354 \pm 52$ & $304 \pm 11$ & $345 \pm 64$ & $341 \pm 47$ & $<0.001$ \\
\hline Septal VA, ms & $20 \pm 21$ & $198 \pm 82$ & $144 \pm 40$ & $114 \pm 91$ & $134 \pm 82$ & $<0.001$ \\
\hline A perturbed, $\%$ & 0 & 0 & 100 & 100 & 53.3 & $<0.001$ \\
\hline A reset with or before SVE reset, $\%$ & 0 & 0 & 100 & 50.0 & 20.0 & $<0.001$ \\
\hline Fusion, $\%$ & 0 & 0 & 50.0 & 50.0 & 6.7 & $<0.001$ \\
\hline Anterograde His/SVE capture, $\%$ & 0 & 0 & 50.0 & 25.0 & 0 & 0.009 \\
\hline SA-VA $(<85 \mathrm{~ms}, \%)$ & $133 \pm 30(0)$ & $160 \pm 42(0)$ & $26 \pm 6(100)$ & $45 \pm 57(100)$ & $71 \pm 56(73.3)$ & $<0.001$ \\
\hline PPI-TCL (<115 ms, \%) & $172 \pm 45(0)$ & $177 \pm 43(0)$ & $92 \pm 29(75.0)$ & $103 \pm 57(77.8)$ & $115 \pm 57(40.0)$ & $<0.001$ \\
\hline cPPI-TCL (<110 ms, \%) & $158 \pm 37(0)$ & $173 \pm 42(0)$ & $55 \pm 22(100)$ & $59 \pm 62(100)$ & $88 \pm 60(80.0)$ & $<0.001$ \\
\hline His-refractory PVS resetting, $\%$ & 0 & 0.0 & 100 & 100 & 80.0 & $<0.001$ \\
\hline PVS PPI-TCL (<115 ms, \%) & $155 \pm 27(0)$ & $125 \pm 37(0)$ & $50 \pm 11(100)$ & $62 \pm 53(100)$ & $88 \pm 51(75.0)$ & $<0.001$ \\
\hline PVS cPPI-TCL $(<110 \mathrm{~ms}, \%)$ & $147 \pm 28(0)$ & $124 \pm 33(0)$ & $24 \pm 25(100)$ & $48 \pm 57(100)$ & $80 \pm 54(91.7)$ & $<0.001$ \\
\hline
\end{tabular}

Abbreviations: A atrium, $A V N R T$ AV nodal reentrant tachycardia, $c P P I$ corrected post-pacing interval, $O R T$ orthodromic reentrant tachycardia, $S A$ stimulus-atrium interval, $P P I$ post-pacing interval, $P V S$ premature ventricular stimulation, $S V E$ septal ventricular electrogram, $T C L$ tachycardia cycle length, $V A$ ventriculoatrial interval

\subsection{Analysis of false negative/positive cases}

The frequency of false negative results of pacing maneuvers in diagnosing ORT showed a correlation with AP location. Most of the parameters had increased sensitivity when ORTs with left free wall AP were excluded (Table 2). Therefore, the studied pacing maneuvers have limited sensitivity in the case of SVT involving a left free wall AP. An eccentric CS activation pattern during SVT can be expected in these cases; however, there are exceptions. This was illustrated by one of our 15 patients: a 69-year-old lady without any previous cardiac interventions, presented with a left free wall AP showing a concentric CS activation pattern during ORT. In the case of this patient, pacing maneuvers were false negative from the RVA suggesting AVNRT (Fig. 1.A and B). However, after repositioning the pacing catheter to the base of the right ventricular
Table 2 Intraclass correlation coefficient, positive predictive value, sensitivity, and specificity of different criteria to diagnose atrioventricular reentrant tachycardia

\begin{tabular}{lllll}
\hline & ICC & Specificity $(\%)$ & \multicolumn{2}{l}{ Sensitivity $(\%)$} \\
\cline { 5 - 5 } & & & For ORT & For ORT excluding left free wall APs \\
\hline A perturbed & 0.66 & 96.3 & 77.4 & 100 \\
A reset with or before SVE & 0.61 & 100 & 41.9 & 62.5 \\
Fusion & 0.51 & 100 & 29.0 & 50.0 \\
Anterograde His/SVE capture & 0.32 & 100 & 16.1 & 31.3 \\
Reset by His-refractory PVS & 0.93 & 96.0 & 74.2 & 87.5 \\
SA-VA $<85$ ms & 0.95 & 100 & 77.4 & 81.3 \\
PPI-TCL $<115$ ms & 0.94 & 100 & 51.6 & 62.5 \\
cPPI-TCL $<110$ ms & 0.93 & 100 & 80.6 & 87.5 \\
PPI-TCL after PVS $<115$ ms & 0.93 & 100 & 82.8 & 87.5 \\
cPPI-TCL after PVS $<110 \mathrm{~ms}$ & 0.94 & 100 & 89.3 & 87.5 \\
\hline
\end{tabular}

Abbreviations: A atrium, $A P$ accessory pathway, $c P P I$ corrected post-pacing interval, $I C C$ interclass correlation, $O R T$ orthodromic reentrant tachycardia, $S A$ stimulus-atrium interval, $P P I$ post-pacing interval, $P P V$ positive predictive value, $P V S$ premature ventricular stimulation, $S V E$ septal ventricular electrogram, $T C L$ tachycardia cycle length, $V A$ ventriculoatrial interval 

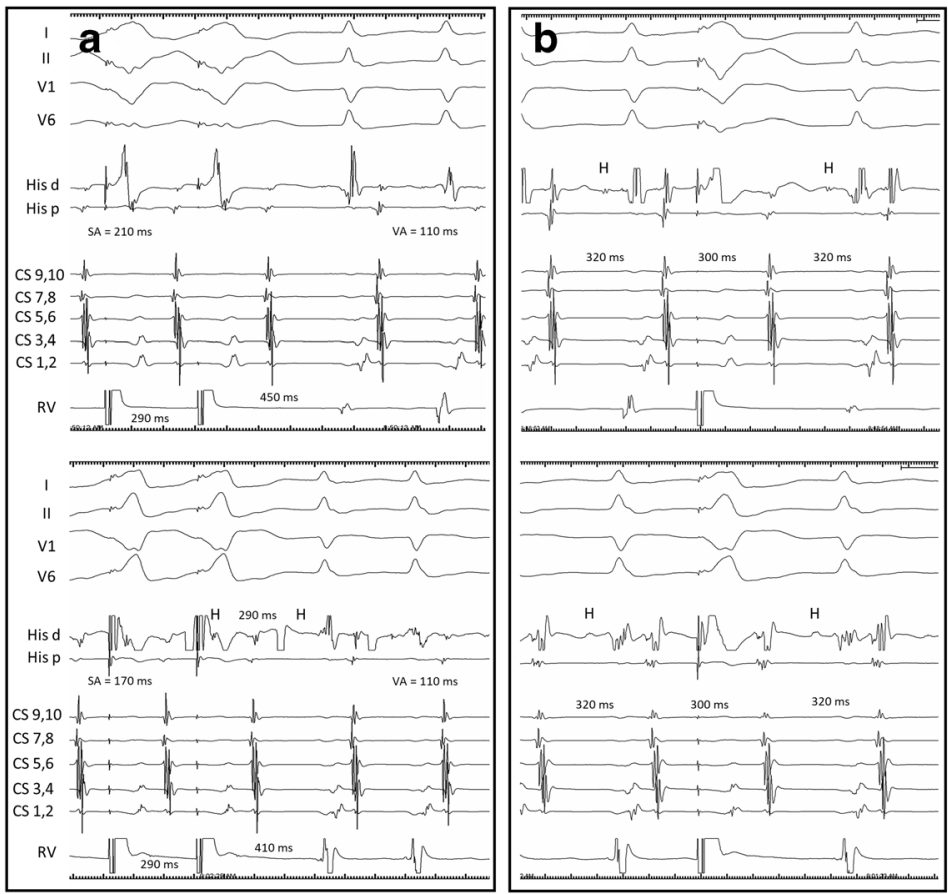
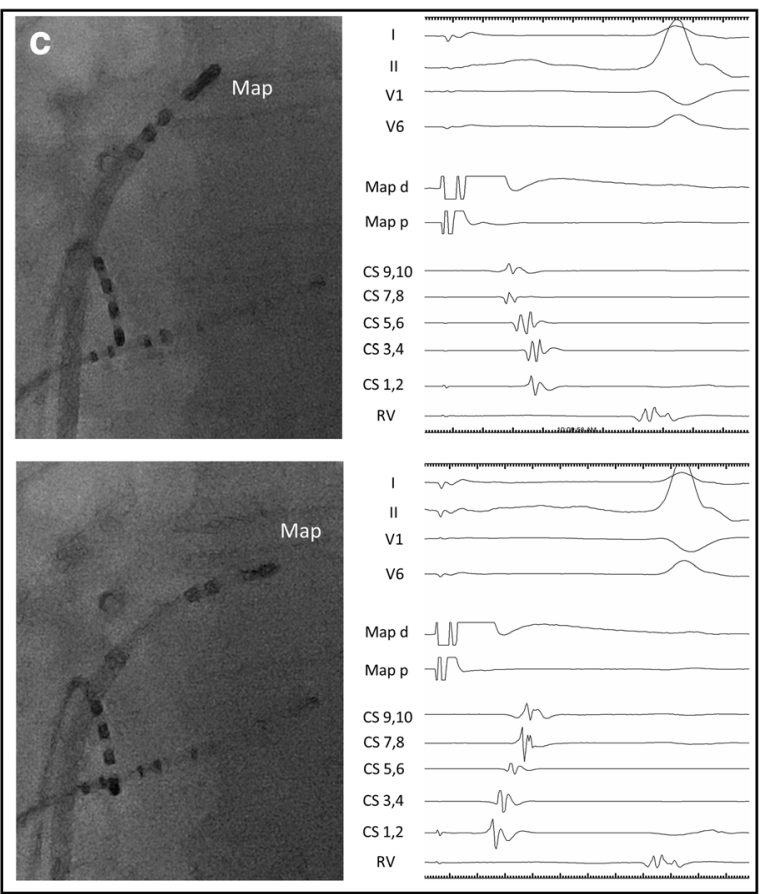

Fig. 1 Surface ECG leads I, II, $V_{1}$, and $V_{6}$, together with intracardiac recordings from the His bundle region (His d and His p), proximal to distal coronary sinus (CS 9,10 - CS 1,2), and right ventricle (RV). Ventricular overdrive pacing (A) and premature ventricular stimulation (B) from the right ventricular apex (top) and base (bottom) in a case of orthodromic reentry tachycardia involving a left free wall accessory pathway (AP), but with central coronary sinus activation. The post-pacing interval, SA-VA value, and the prematurity required for resetting

outflow tract, the maneuvers were positive for ORT (Fig. 1.A and B). Transseptal mapping revealed fragmented atrial electrograms and delayed conduction in the vicinity of the AP promoting concentric CS activation during ORT (Fig. 1.C).

While false negative PPI was corrected in most ORTs by subtracting AV nodal delay (cPPI), 2 of 4 ORTs with a slowly conducting septal AP showed cPPI and SA-VA values above the cutoff after VOP and PVS.

False positivity of PVS was encountered in one case. This was a 37-year-old woman with a long-RP tachycardia. The tachycardia was induced by ventricular extrastimulation with an A-A-(H)-V response, suggesting atrial tachycardia (AT) (Fig. 2.B); however, after VOP, we observed an A-(H)-V response excluding AT. PVS delivered during His refractoriness reproducibly delayed the next atrial activation and reset the tachycardia (Fig. 2.C), suggesting participation of a retrogradely conducting AP. However, several features argued against ORT. The AH interval, during SVT, was paradoxically shorter than during sinus rhythm (Fig. 2.A and B), pointing to the AH being a pseudointerval during tachycardia $[13,16]$. The SA-VA, HA ${ }_{V O P}-H_{S V T}[14]$, PPI, and cPPI values were all much longer than published limits for ORT (Fig. 2.D and E) and well exceeded the upper limit suggested for ORT using a slowly conducting AP (125 ms) [13]. At the beginning of
( $50 \mathrm{~ms}$ before vs. on time with the anticipated His activation) are much greater from the apex vs. the base of the right ventricle. Anterograde His capture $(\mathrm{H})$ is only seen with basal pacing. Left atrial activation delay at the AP insertion site demonstrated by markedly different CS activation patterns when pacing from (concentric CS pattern) vs. slightly lateral (eccentric CS activation) to the AP insertion (C). The location of the mapping catheter (Map) is marked on fluoroscopic images acquired in the left anterior oblique projection

VOP, the His bundle is captured retrogradely and is evidenced to be dissociated from the tachycardia also ruling out ORT where the His bundle is part of the circuit (Fig. 2.D)[17].

Therefore, the diagnosis of atypical AVNRT was made and ablation at the slow pathway region, eliciting junctional acceleration eliminated the SVT. The finding of an A-A- $(\mathrm{H})-\mathrm{V}$ response upon induction from the ventricle and Hisrefractory PVC resetting the tachycardia were interpreted as a result of a bystander, concealed nodo-fascicular/nodo-ventricular (NF/NV) AP.[13, 16] A retrograde dual (1:2) atrial response (conduction over both the fast pathway and NF/NV AP) explains the findings upon ventricular induction, while insertion of the NF/NV AP into the slow AV nodal pathway and delayed conduction of the premature stimulus explains the postexcitation by the His-refractory PVC, according to Ho et al.[13]

\section{Discussion}

According to the present study, the main limitations of ventricular pacing maneuvers in differentiating ORT from AVNRT are: 


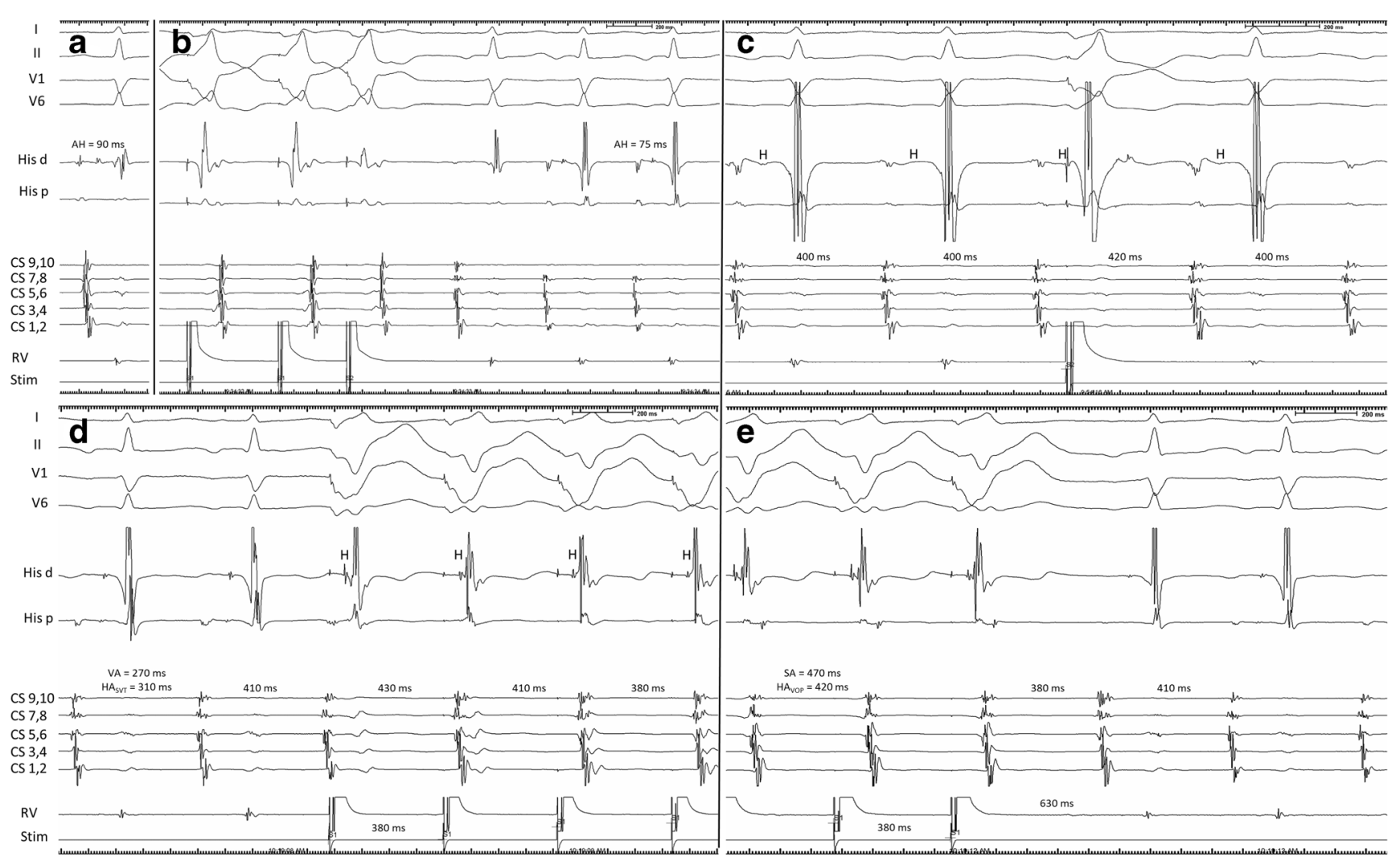

Fig 2 The atrial to His (AH) interval during sinus rhythm (A) is paradoxically longer than the AH during tachycardia (B). The tachycardia is induced by a retrograde dual atrial response to the ventricular extrastimulus (B). Ventricular premature stimulation coinciding with the His bundle depolarization $(\mathrm{H})$ reproducibly delays the next atrial activation and resets tachycardia (C). Ventricular overdrive pacing during tachycardia immediately entrains the His bundle as evidenced by retrograde His potentials $(\mathrm{H})$ at the paced cycle length. His bundle activation is dissociated from the tachycardia which becomes entrained after the third ventricular paced complex. The first paced ventricular beat delays atrial activation similarly to that in panel C (D). Immediately after VOP a "pseudo $\operatorname{VAA}(\mathrm{H}) \mathrm{V}$ " response is seen due to the SA interval exceeding the paced cycle length (E). The post-pacing interval and the SA-VA and $\mathrm{HA}_{\mathrm{VOP}}-\mathrm{HA}_{\mathrm{SVT}}$ values are long (D and $\mathrm{E}$ ). The above findings were interpreted to be the result of atypical AVNRT with a bystander, concealed nodo-fascicular/nodo-ventricular AP. Recording channels are the same as on Fig. 1. See text for further discussion

Some of the features of VOP and PVS examined in this study were originally described only in AVRT involving a septal AP [5, 7-9], while others were tested also in the case of free wall APs $[3,4,6,10]$.

We intended to provide an independent evaluation of all ventricular pacing maneuvers and their limitations, that also has relevance to cases without right and left atrial recording.

\subsection{Semi-quantitative parameters of VOP}

At the beginning of VOP, the pacing train gradually catches up with anterograde ventricular activation from the SVT circuit, producing variable degrees of fusion of ventricular complexes until a stable QRS is established. This so-called transition zone offers valuable diagnostic information for differentiating ORT from AVNRT. Perturbation of atrial rate in the transition zone [6], and atrial reset simultaneously with or before advancement of the basal, septal ventricle [8] are consistent with ORT. After the transition zone, during stable 
entrainment by VOP, the stimulated wavefront collides with the anterograde wavefront of the previous beat. In the case of ORT, this collision can occur in ventricular myocardium leading to manifest QRS fusion (manifest entrainment).[7] However, in some cases of AVRT and all cases of AVNRT, collision occurs above the ventricles; thus, QRS fusion is not present (concealed entrainment). On a similar principle, intracardiac fusion is evidenced when the His potential of the first return beat after ventricular entrainment is captured anterogradely, by the orthodromic wavefront, signifying ORT.[5]

These diagnostic criteria require the recognition of surface (transition zone, fusion) or intracardiac (SVE, His potential during VOP) ECG patterns which may be ambiguous in some cases. This likely explains the finding of poor agreement between observers blinded to the ultimate diagnosis in this study. Nevertheless, when these criteria were recognized in 16-77\% of ORT cases, they represented true positivity for ORT, resulting in $100 \%$ specificity.

\subsection{Quantitative parameters of VOP and PVS}

The measurement of certain intervals during and after VOP/ PVS was found to be more reproducible between observers.

During ORT and entrainment of ORT, the activation of the atrium and the ventricle happens in series. In contrast, in the case of AVNRT, the atrium and the ventricle are activated simultaneously. As during entrainment of AVNRT this simultaneous activation changes to serial, a difference $(>85 \mathrm{~ms})$ develops between VA intervals during SVT compared to entrainment (SA-VA).[9]

The PPI after entrainment by VOP or resetting by PVS is related to the proximity of the pacing site to the reentry circuit.[9] However, the PPI can increase due to ratedependent conduction slowing in the circuit during pacing. In ORT, conduction slowing can occur in the AV node or a slowly conducting AP. In the first case, the AH interval following the last entrained cycle is prolonged causing prolonged PPI (and PPI-TCL). As described by González-Torrecilla et al. [10] and confirmed in this series, subtracting the increment in AV nodal conduction time in the first PPI from the PPI-TCL difference (cPPI-TCL) improves diagnostic accuracy of this maneuver. Rate-dependent AV nodal delay was less of a problem after single PVS resetting of SVT, resulting in better sensitivity even without correction. However, similar to the findings of Bennett et al. slowly conducting, septal APs were associated with a $50 \%$ rate of false negativity due to ratedependent slowing in AP conduction during pacing.[18]

\subsection{His-refractory PVS}

In AVNRT without a retrogradely conducting, bystander AP-when PVS is delivered in His bundle refractoriness- the stimulated wavefront collides with the tachycardia wavefront below the level of the His; thus, resetting of reentry cannot occur. In contrast, during ORT, His-refractory PVS might elicit atrial preexcitation and resetting of the reentry circuit through the AP.[19] A classic teaching point is that this response does not necessarily prove the participation of the $\mathrm{AP}$ in the tachycardia, which can still be a bystander during AVNRT or AT.[20] Traditionally, it has been accepted that the delay or termination of SVT by His-refractory PVS on the other hand proves ORT.[21] In recent years, however several publications suggested that a concealed NF/NV AP inserting into the AV nodal slow pathway can result in such a response during atypical AVNRT.[13, 16, 22-24] Diagnostic criteria have been developed for the differentiation of ORT (involving an atrioventricular or a NF/NV AP) and atypical AVNRT with a bystander, concealed NF/NV AP. [25, 26] The latter mechanism was responsible for the only false positive result of PVS in our series.

\subsection{Impact of AP location on pacing maneuvers}

Sensitivity for ORT was better for right free wall and septal APs, compared to left free wall APs (Table 2) This is related to the pacing electrode being farther from the ORT circuit for left free wall APs. Repositioning of the pacing catheter closer to the AP improved diagnostic accuracy (Fig. 1). It may be argued that ORT involving a left free wall AP should present with an eccentric CS activation pattern making it easy to differentiate from AVNRT. However, concentric CS activation by a left free wall AP has been reported after previous ablation [1] and it can also occur spontaneously, without a previous cardiac intervention as seen in one patient in this series (Fig. 1). Moreover, an eccentric pattern does not exclude AVNRT.[2]

Therefore, it is important to bear in mind that the pacing maneuvers developed for differentiating ORT from AVNRT might be misleading when the pacing catheter is far from the ORT circuit, as in the case of a left free wall AP. Performing the maneuvers closer to the site of the presumed AP improves diagnostic accuracy in ambiguous cases.

\subsection{Limitations of the study}

The number of patients that could be enrolled in the study was limited by the fact that not all ventricular pacing maneuvers are routinely performed in all SVTs and when attempted they can be hampered by spontaneous oscillation in cycle length, inability to entrain/reset, or termination of the tachycardia. Since one or more ventricular pacing maneuvers were systematically omitted in a considerable number of patients (e.g., those with typical AVNRT or ORT with left AP), therefore patient inclusion was tilted towards atypical AVNRT and septal ORT. While this can strengthen the results by 
overrepresentation of the diagnostically more challenging long-RP SVTs and septal APs, deviation from the distribution of SVT mechanisms in the general population precludes defining the true frequency with which the presented pitfalls are likely to be encountered in routine practice.

\subsection{Recommended approach to differential diagnosis}

When confronted by the task to differentiate ORT from AVNRT in the EP lab, reliance on a single parameter is not sufficient. Performance of atrial, ventricular, and para-Hisian pacing during sinus rhythm before induction of tachycardia and examining features at the initiation and commencement of SVT provide important information aiding in correctly diagnosing the mechanism. Ventricular pacing maneuvers during SVT are useful for further refinement if the TCL is sufficiently stable. During VOP, unequivocally observing one or more semi-quantitative features strongly suggests ORT, their absence however is not decisive. Quantitative measurements, with appropriate correction for AV nodal slowing, and Hisrefractory PVS are more reproducible and sensitive. However, when values are close to the cutoff repositioning of the pacing catheter to the right or left ventricular base and repeating the maneuvers is recommended.

\section{Conclusion}

No single maneuver is $100 \%$ sensitive in the differential diagnosis of ORT vs. AVNRT. We identified specific weaknesses in the case of all ventricular pacing maneuvers. Semiquantitative features of VOP are less reproducible among observers. We demonstrated that better reliability and diagnostic accuracy are provided by quantitative measurements of VOP and PVS and His-refractory PVS. However, ORT circuits remote from the ventricular pacing catheter, rate-dependent conduction slowing with pacing, and a concealed, bystander NF/NV AP during atypical AVNRT are important caveats to bear in mind. Based on the understanding of these pitfalls, further diagnostic steps can be pursued in ambiguous cases.

\section{Declarations}

Conflict of interest The authors declare no competing interests.

\section{References}

1. Pap R, Sághy L. Conflicting results of pacing maneuvers during supraventricular tachycardia. PACE - Pacing Clin Electrophysiol. 2012;35:1392-4.

2. Hwang C, Martin DJ, Goodman JS, Gang ES, Mandel WJ, Swerdlow CD, et al. Atypical atrioventricular node reciprocating tachycardia masquerading as tachycardia using a left-sided accessory pathway. J Am Coll Cardiol. 1997;30:218-25.

3. Knight BP, Ebinger M, Oral H, Kim MH, Sticherling C, Pelosi F, et al. Diagnostic value of tachycardia features and pacing maneuvers during paroxysmal supraventricular tachycardia. J Am Coll Cardiol. 2000;36:574-82.

4. Kaiser DW, Hsia HH, Dubin AM, Liem LB, Viswanathan MN, Zei $\mathrm{PC}$, et al. The precise timing of tachycardia entrainment is determined by the postpacing interval, the tachycardia cycle length, and the pacing rate: theoretical insights and practical applications. Heart Rhythm. 2016;13:695-703.

5. Nagashima K, Kumar S, Stevenson WG, Epstein LM, John RM, Tedrow UB, et al. Anterograde conduction to the His bundle during right ventricular overdrive pacing distinguishes septal pathway atrioventricular reentry from atypical atrioventricular nodal reentrant tachycardia. Heart Rhythm. 2015;12:735-43.

6. Al Mahameed ST, Buxton AE, Michaud GF. New criteria during right ventricular pacing to determine the mechanism of supraventricular tachycardia. Circ Arrhythm Electrophysiol. 2010;3:578-84.

7. Ormaetxe JM, Almendral J, Arenal A, Martínez-Alday JD, Pastor A, Villacastín JP, et al. Ventricular fusion during resetting and entrainment of orthodromic supraventricular tachycardia involving septal accessory pathways: implications for the differential diagnosis with atrioventricular nodal reentry. Circulation. 1993;88:262331. https://doi.org/10.1161/01.CIR.88.6.2623.

8. Calvo D, Ávila P, García-Fernández FJ, Pachón M, Bravo L, Eidelman G, et al. Differential responses of the septal ventricle and the atrial signals during ongoing entrainment: a method to differentiate orthodromic reciprocating tachycardia using septal accessory pathways from atypical atrioventricular nodal reentry. Circ Arrhythm Electrophysiol. 2015;8:1201-9.

9. Michaud GF, Tada H, Chough S, Baker R, Wasmer K, Sticherling $\mathrm{C}$, et al. Differentiation of atypical atrioventricular node re-entrant tachycardia from orthodromic reciprocating tachycardia using a septal accessory pathway by the response to ventricular pacing. J Am Coll Cardiol. 2001;38:1163-7.

10. González-Torrecilla E, Arenal A, Atienza F, Osca J, GarcíaFernández J, Puchol A, et al. First postpacing interval after tachycardia entrainment with correction for atrioventricular node delay: a simple maneuver for differential diagnosis of atrioventricular nodal reentrant tachycardias versus orthodromic reciprocating tachycardias. Heart Rhythm. 2006;3:674-9.

11. Veenhuyzen GD, Coverett K, Quinn FR, Sapp JL, Gillis AM, Sheldon R, et al. Single diagnostic pacing maneuver for supraventricular tachycardia. Heart Rhythm. 2008;5:1152-8.

12. Javier García-Fernandez F, Almendral J, Pachón M, GonzálezTorrecilla E, Martín J, Gallardo R. Differentiation of atrioventricular nodal reentrant tachycardia from orthodromic reciprocating tachycardia by the resetting response to ventricular extrastimuli: comparison to response to continuous ventricular pacing. J Cardiovasc Electrophysiol. 2013;24:534-41.

13. Ho RT, Frisch DR, Pavri BB, Levi SA, Greenspon AJ. Electrophysiological features differentiating the atypical atrioventricular node - dependent long RP supraventricular tachycardias. Circ Arrhythm Electrophysiol. 2013;6:597-605.

14. Ho RT, Mark GE, Rhim ES, Pavri BB, Greenspon AJ. Differentiating atrioventricular nodal reentrant tachycardia from atrioventricular reentrant tachycardia by $\triangle H A$ values during entrainment from the ventricle. Heart Rhythm. 2008;5:83-8.

15. Nakagawa H, Jackman WM. Para-Hisian pacing: useful clinical technique to differentiate retrograde conduction between accessory atrioventricular pathways and atrioventricular nodal pathways. Heart Rhythm. 2005;2:667-72.

16. Ho RT, Fischman DL. Entrainment versus resetting of a long RP tachycardia: what is the diagnosis? Heart Rhythm. 2012;9:312-4. 
17. Ho RT, Levi SA. An atypical long RP tachycardia - what is the mechanism? Heart Rhythm. 2013;10:1089-90.

18. Bennett MT, Leong-Sit P, Gula LJ, Skanes AC, Yee R, Krahn AD, et al. Entrainment for distinguishing atypical atrioventricular node reentrant tachycardia from atrioventricular reentrant tachycardia over septal accessory pathways with long-rp tachycardia. Circ Arrhythm Electrophysiol. 2011;4:506-9.

19. Sellers TD Jr, Gallagher JJ, Cope GD, Tonkin AMWA. Retrograde atrial preexcitation following premature ventricular beats during reciprocating tachycardia in the Wolff-Parkinson-White syndrome. Eur J Cardiol. 1977;4:283-94.

20. Posan E, Gula LJ, Skanes AC, Krahn AD, Yee R, Klein GJ. A classic but uncommon principle. J Cardiovasc Electrophysiol. 2006;17:106-7.

21. Bardy GH, Packer DL, German LD, Coltorti F, Gallagher JJ. Paradoxical delay in accessory pathway conduction during long $\mathrm{R}-\mathrm{P}^{\prime}$ tachycardia after interpolated ventricular premature complexes. Am J Cardiol. 1985;55:1223-5.

22. Bansal S, Berger RD, Spragg DD. An unusual long RP tachycardia: what is the mechanism? Heart Rhythm. 2015;12:845-6.
23. Vaseghi M, Fujimura O, Shivkumar K. Narrow QRS complex tachycardia: what is the mechanism? Heart Rhythm. 2013;10: 1402-4.

24. Boonyapisit W, Kuhne M, Morady F, Jongnarangsin K. Supraventricular tachycardia with delayed atrial depolarization after His-synchronous ventricular stimulation: what is the mechanism? Heart Rhythm. 2010;7:280-1.

25. Soares Correa F, Lokhandwala Y, Sánchez-Quintana D, Mori S, Anderson RH, Wellens HJJ, et al. Unusual variants of pre-excitation: from anatomy to ablation: part III - clinical presentation, electrophysiologic characteristics, when and how to ablate nodo-ventricular, nodo-fascicular, fasciculoventricular pathways, along with considerations of permanent. J Cardiovasc Electrophysiol. 2019;30: 3097-115.

26. Ho R, Ortman M, Levi S. The various manifestations of concealed nodo-fascicular/nodo-ventricular bypass tracts. Heart Rhythm. 2020;17:1280-90

Publisher's note Springer Nature remains neutral with regard to jurisdictional claims in published maps and institutional affiliations. 